\title{
The pyruvate dehydrogenase kinase 2 (PDK2) is associated with conidiation, mycelial growth, and pathogenicity in Fusarium graminearum
}

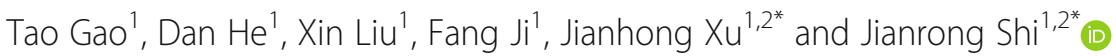

\begin{abstract}
Pyruvate dehydrogenase kinase (PDK) is a mitochondrial enzyme in a variety of eukaryotes, including the plant pathogen Fusarium graminearum. This enzyme can reduce the oxidation of glucose to acetyl-coA by phosphorylation and selectively inhibits the activity of pyruvate dehydrogenase (PDH), which is a kind of pyruvate dehydrogenase complex (PDC). In this study, we investigated the F. graminearum pyruvate dehydrogenase kinase encoded by FgPDK2, which is a homologue of Neurospora crassa PDK2. The disruption of the FgPDK2 gene led to several phenotypic defects including effects on mycelial growth, conidiation, pigmentation, and pathogenicity. The mutants also showed decreased resistance to osmotic stress and cell membrane/wall-damaging agents. The FgPDK2 deletion mutant exhibited reduced virulence. All of these defects were restored by genetic complementation of the mutant with the complete FgPDK2 gene. Overall, the results demonstrated that FgPDK2 is crucial for the growth of F. graminearum and can be exploited as a potential molecular target for novel fungicides to control Fusarium head blight caused by F. graminearum.
\end{abstract}

Keywords: Fusarium graminearum, Pyruvate dehydrogenase kinase, Vegetative differentiation, Reactive oxygen species (ROS)

\section{Introduction}

Fusarium head blight (FHB) caused by Fusarium species is an economically devastating disease of wheat and other small grain cereal crops around the world (Mcmullen et al. 1997). The toxin produced by F. graminearum also leads to a great risk to human and animal health and food safety (Kleter and Marvin 2009). Despite the high economic impact of FHB, there is currently no

\footnotetext{
*Correspondence: xujianhongnj@126.com; jianrong63@126.com

1 Jiangsu Key Laboratory for Food Quality and Safety-State Key Laboratory Cultivation Base, Ministry of Science and Technology/Key Laboratory for Control Technology and Standard for Agro-product Safety and Quality, Ministry of Agriculture and Rural Affairs/Key Laboratory for Agro-product Safety Risk Evaluation (Nanjing), Ministry of Agriculture and Rural Affairs/ Collaborative Innovation Center for Modern Grain Circulation and Safety/ Institute of Food Safety and Nutrition, Jiangsu Academy of Agricultural Sciences, Nanjing 210014, Jiangsu, China

Full list of author information is available at the end of the article
}

effective management strategy that does not rely on fungicides. Therefore, a better understanding of fungal development is helpful to develop new fungicides and new effective strategies to control FHB, which is important for wheat production and safety.

In general, biological cells continuously monitor their internal and external environments and sense signals for proliferation, differentiation, stagnation, or death, incorporating many exogenous foreign signals to control the possible progression or suspension of some or all cell activity. In eukaryotes, when the external environment changes or internal growth and development begin, the phosphorylation-dephosphorylation cycle is the main mechanism by which pathways are changed (Dickman and Yarden 1999). In most organisms, pyruvate is important as the end product of glycolysis (Papagianni

(c) The Author(s). 2020 Open Access This article is licensed under a Creative Commons Attribution 4.0 International License, which permits use, sharing, adaptation, distribution and reproduction in any medium or format, as long as you give

appropriate credit to the original author(s) and the source, provide a link to the Creative Commons licence, and indicate if changes were made. The images or other third party material in this article are included in the article's Creative Commons licence, unless indicated otherwise in a credit line to the material. If material is not included in the article's Creative Commons licence and your intended use is not permitted by statutory regulation or exceeds the permitted use, you will need to obtain permission directly from the copyright holder. To view a copy of this licence, visit http://creativecommons.org/licenses/by/4.0/. 
2012) and can be metabolized by the pyruvate dehydrogenase complex (PDC). In mammals, the PDC can catalyze pyruvate metabolism to generate acetyl CoA through pyruvate decarboxylation; the main components of the PDC include three enzymes: pyruvate dehydrogenase (PDH, E1), dihydrothiocinamide acetyl transferase (E2) and dihydrothiocinamide dehydrogenase (E3) (Sugden and Holness 2011). The regulation of PDC activity is mainly achieved through the phosphorylation (inhibition) and dephosphorylation (activation) of serine residues at sites 293, 300 and 232 of the E1 alpha subfamily in PDH (Dickman and Yarden 1999). Pyruvate dehydrogenase kinase (PDK) is a kind of mitochondrial enzyme that can reduce the oxidation of glucose to acetyl-coA by phosphorylation and selectively inhibits the activity of PDH. At the same time, this step causes an increase in glycolysis and affects the oxidation of glucose (Roche and Hiromasa 2007). The research on PDK in mammals is extensive, and the activity of PDH complex enzymes can directly regulate the addition of pyruvate in the tricarboxylic acid cycle. In Saccharomyces cerevisiae, the conversion of pyruvate to acetyl-coA is accomplished by two PDKs. One regulates the phosphorylation of Pda1p ser133 residues, and the other regulates the phosphorylation of alpha subunits in PDCs (Krause-Buchholz et al. 2006). There are two PDHKs (Pkp1p and Pkp2p) in S. cerevisiae and deletion of them results in defects during growth in the presence of acetate and ethanol as carbon sources (Steensma et al. 2008). This phenomenon indicates that the PDHKs in $S$. cerevisiae may cause a predicted aborted carbon utilization cycle (Steensmays 1996). The PDHK in $A$. nidulans, PkpC (AN6207) was shown to be important for the secretion of cellulose and cellulose in the process of growth (Ries et al. 2018).

In F. graminearum, there are two PDKs (PDK1 and PDK2) with sequence similarity to the $S$. cerevisiae PDH kinases, sharing 26 and $29 \%$ amino acid identity with PKP1 and PKP2, respectively. The deletion of FgPDK1 causes reduced growth on minimal medium supplemented with sucrose. The fungal morphology, conidiation and pathogenicity of FgPDK1 deletion mutants also changed greatly compared with those of the wild-type strain (Gao et al. 2016). The objective of this study was therefore to explore the biological functions of another putative PDH kinase, FgPDK2. Genetic evidence indicated that FgPDK2 is essential for fungal growth and sexual reproduction, and fungal pathogenesis.

\section{Materials and methods}

\section{Strains and culture conditions}

The parental strain used for generating deletion mutants was $\mathrm{PH}-1$, which is the standard strain of F. graminearum (Wang et al. 2019). Three different kinds of media (PDA, MM and $\mathrm{CM}$ ) were used to analyze the colony morphology, and mycelial growth was measured with the cross-crossing method previously described (Gao et al. 2016). To evaluate the role of the FgPDK2 gene in conidia production capacity, we inoculated ten 5-mm plugs of mycelia growth on PDA (3-day-old colony) into $100 \mathrm{ml} \mathrm{MBB}$ liquid medium (1.5\% mung bean) with shaking at $180 \mathrm{rpm}$ at $25^{\circ} \mathrm{C}$ for 5 days (Chen and Zhou 2009). The capacity of conidia germination was analysed with the medium of YEPD ( $1 \%$ yeast extract, $2 \%$ peptone, $2 \%$ glucose). The medium used for fungal RNA mycelium sample collection was GYEP (5\% glucose, $0.1 \%$ yeast extract, and $0.1 \%$ peptone) (Zheng et al. 2014).

\section{Sequence analysis}

The BLASTP algorithm was used for querying the sequence of the PKP2 gene in Saccharomyces cerevisiae S288C (NCBI accession number NP_011456.5). The PKP2 gene was used as the homologous sequence for searching the sequence of the putative mitochondrial protein kinase gene FgPDK2 (FGSG_07381.3) in the database of F. graminearum (http://fungidb.org/fungidb/). Clustal W was used for the orthologues aligned of PDK2 amino acid sequences in different species. And MEGA v. 4.0.2 software was used for phylogenetic analysis by the method of neighbourjoining (Kumar et al. 2008).

\section{DNA and RNA extraction}

The CTAB (hexadecyl trimethyl ammonium bromide) method was used to extract mycelia genomic DNA (Zhang et al. 2009) and an RNeasy kit (Tian gen, China) was used to extract RNA from the samples that were cultured in YEPD and GYEP for $48 \mathrm{~h}$. A PrimeScript ${ }^{\circ}$ RT reagent kit (TaKaRa) was used to synthesize the first-strand cDNA, and the real-time detection system was a Roche Diagnostics system (Wang et al. 2010).

\section{Deletion of the FgPDK2 gene in F. graminearum}

To analyze the function of $F g P D K 2$, polyethylene glycol (PEG)-mediated protoplast transformation was used to construct the FgPDK2 deletion mutant ( $\triangle$ FgPDK2) with the genetic background of PH-1 (Gao et al. 2016). The strategy of double-joint PCR was used to construct the FgPDK2 gene replacement vector (Yu et al. 2004). First, the $1.0 \mathrm{~kb}$ upstream (1.0-up) and $1.0 \mathrm{~kb}$ downstream (1.0-down) of $F g P D K 2$ gene were amplified from the parent strain $\mathrm{PH}-1$ with the primers $\mathrm{A} 1 / \mathrm{A} 2$ and $\mathrm{A} 3 / \mathrm{A} 4$. Then, the hygromycin resistance gene ( $H P H$-tk cassette) was initially amplified from the PtrpChptA-PItk plasmid with the primers of HTF/HTR. All of the primers are listed in supplementary data Table S1. To complement the full-length $F g P D K 2$ with the deletion mutants, the fragment of gene was inserted into the pYF11 plasmid 


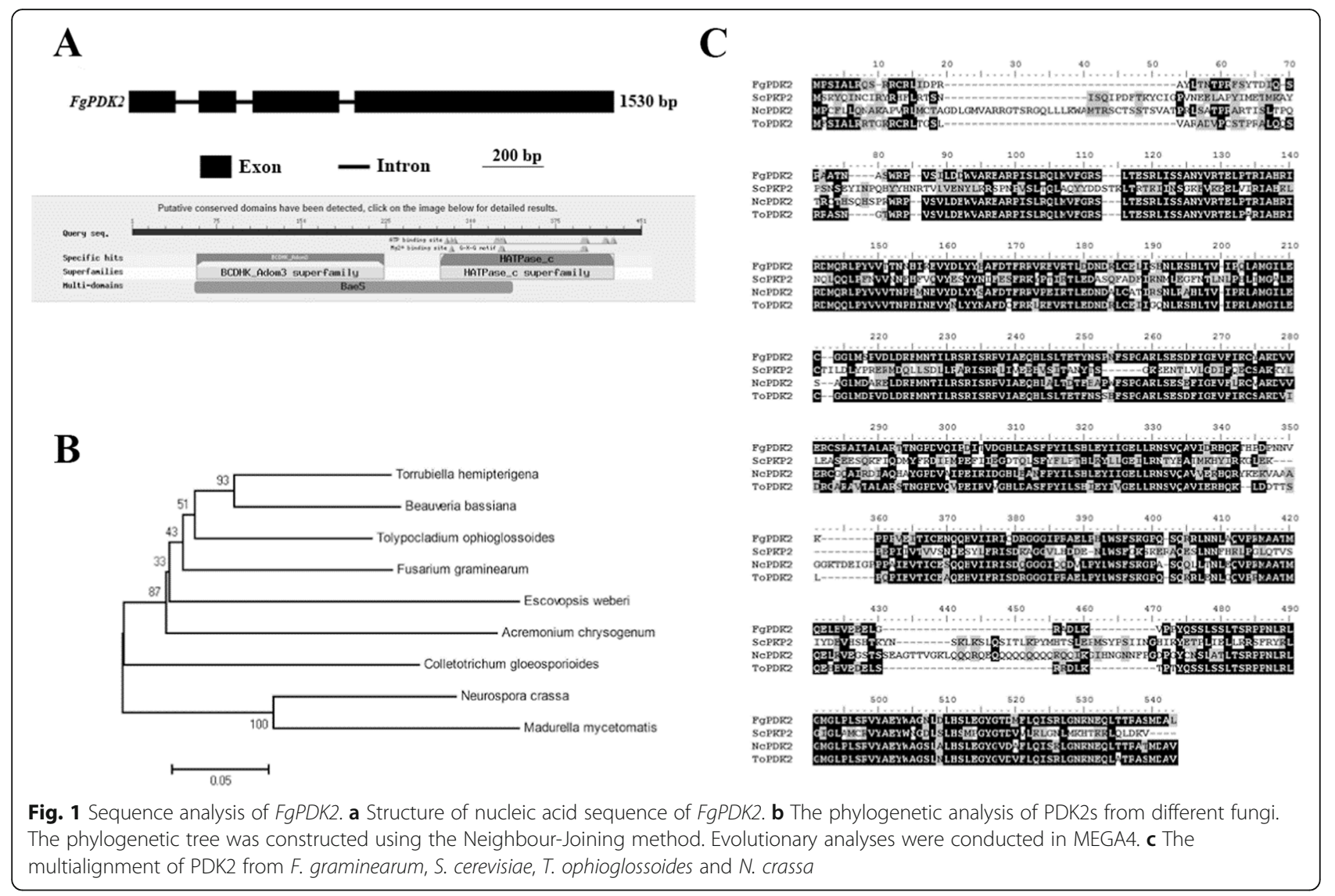

(Bruno et al. 2014), then the recombinant plasmid pYF11-FgPDK2 was transformed into $\triangle$ FgPDK2. An AxyPreTP DNA Gel Extraction Kit (Axygen Biosciences, China) was used to purify the PCR products. Phenotypic characterization was firstly used to screen the resulting transformants, then the results of PCR amplification and Southern blotting confirmed the deletion mutants.

\section{Cellular stress sensitivity evaluation}

To determine the cell sensitivity of different strains, a 5mm (diameter) mycelial plug was placed on PDA plates that were supplemented with the following solutes: osmotic stress inducers $\mathrm{NaCl}$ and $\mathrm{KCl}$; the cell wall inhibitors caffeine and congo red; the cell membrane damager sodium dodecyl sulfate (SDS). The percentage of mycelial radial growth inhibition (RGI) was calculated using a previously described formula (Liu et al. 2019).

\section{DON analysis}

To determine the amount of DON produced by the mutants in vitro, five $5-\mathrm{mm}$ (diameter) mycelial plugs were suspended in GYEP liquid medium and shaken at $28^{\circ} \mathrm{C}$ in the dark for 7 days followed by filtration, drying and measurement of mycelium weight. DON was extracted and analysed using the protocol previously described by Ji et al.
(2014). The HPLC-MS/MS system (Shimadzu 30A L C system coupled to a Triple Quad 6500 plus) was used to determine the quantity of DON with the methods which was previously described by Dong et al. (2016).

To analyze the expression levels of two trichothecene biosynthesis genes, Tri5 and Tri6, ten mycelial plugs (5 $\mathrm{mm}$ diameter) from the edge of a 3-day-old colony of each strain were added into $100 \mathrm{ml}$ GYEP and cultured for $48 \mathrm{~h}$ at $28{ }^{\circ} \mathrm{C}$ in the dark. Total RNA was extracted from the mycelia of each sample, and quantitative realtime PCR assays was used to determine the expression levels of Tri5 and Tri6.

\section{Pathogenicity in fresh tomatoes}

To analyze the effect of FgPDK2 on pathogenicity, mycelia of all strains were inoculated to fresh tomatoes (Di Pietro et al. 2001). After inoculation, the tomatoes were cultivated with $12 \mathrm{~h}$ of light per day for 5 days at $30{ }^{\circ} \mathrm{C}$ followed by measurement of lesions. . Each mutant or strain was represented by three replicate fruits, and the experiment was performed three times.

\section{Statistical analysis}

At least three replicated measurements were used to calculate the mean standard deviation (SD) and data are 


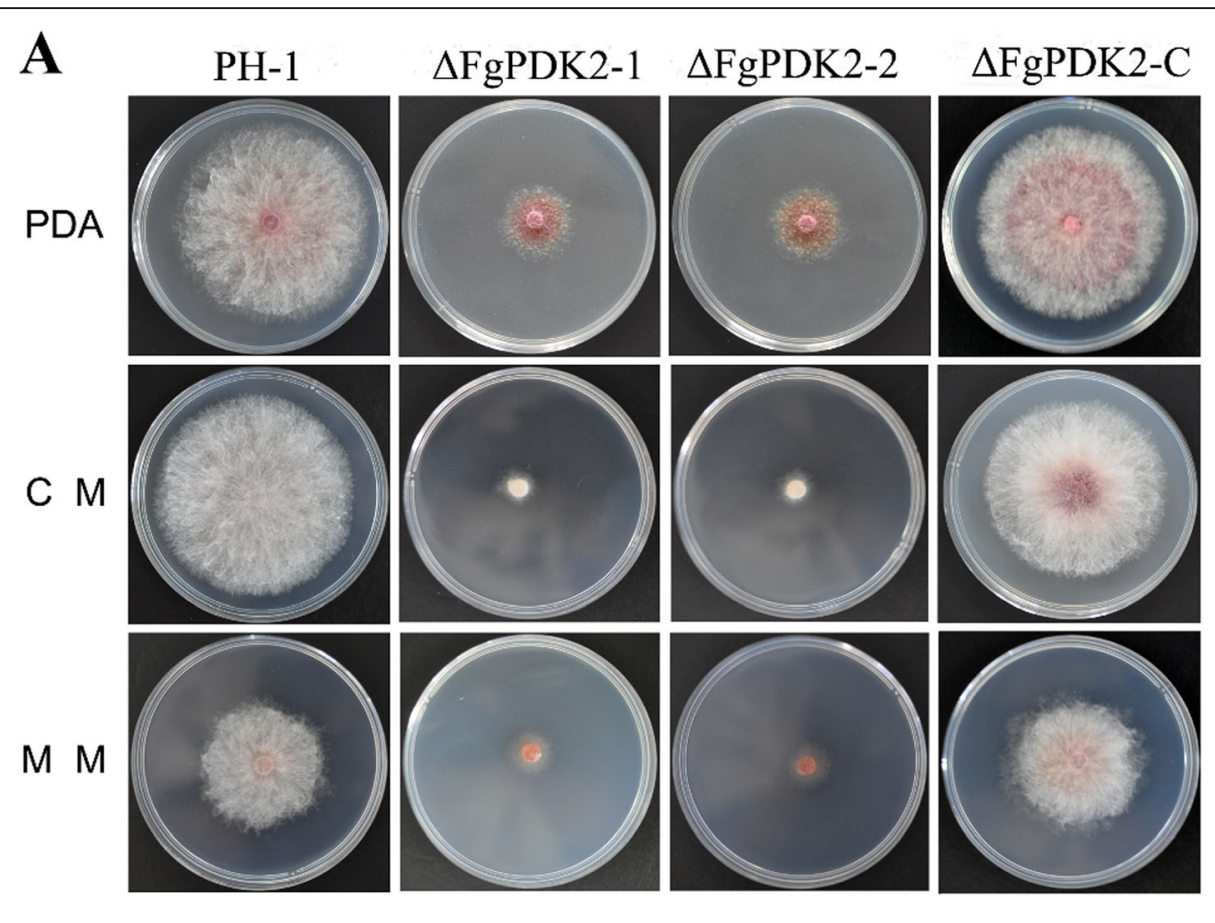

B

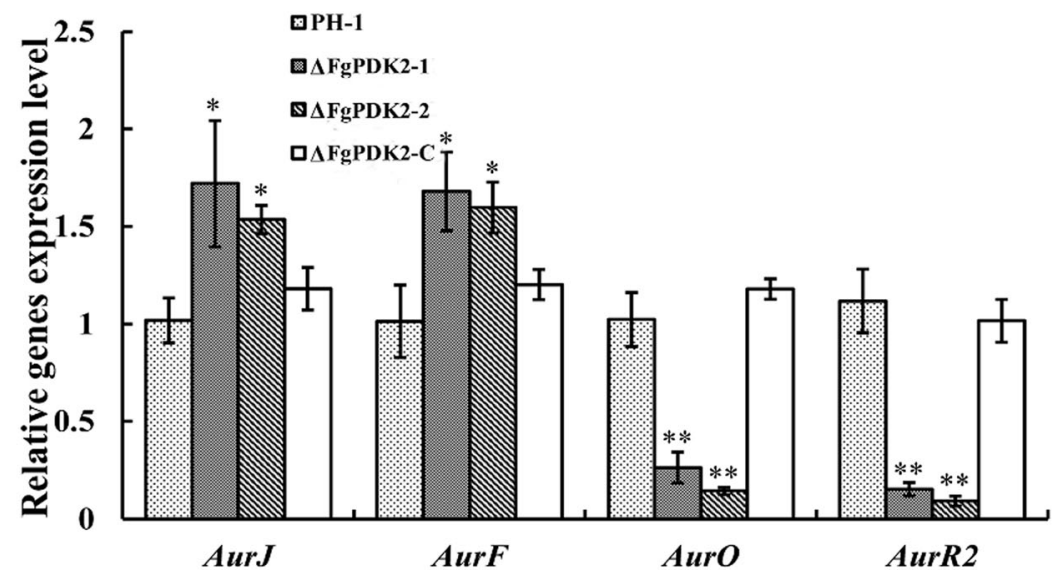

Fig. 2 Effect of FgPDK2 deletion on F. graminearum colony morphology and pigment production. a The wild-type strain (PH-1), FgPDK2 deletion mutant ( $\triangle$ FgPDK2), and complemented strain ( $\triangle$ FgPDK2-C) were grown on PDA, CM, and MM for 4 days at $25^{\circ} \mathrm{C}$. $\mathbf{b}$ Relative expression levels of four pigment-formation genes (FgAurJ, FgAur, FgAurO, and FgAurR2). The error bars in each column represent the standard deviation (SD) from three independent experiments; ${ }^{*} P<0.05$ or ${ }^{*} P<0.01$ by Student's t-test

presented as the mean \pm SD. The statistical comparison between wild-type parent $\mathrm{PH}-1$ and deletion mutants were evaluated by SD of variance (ANOVA) with the software package SPSS 2.0.

\section{Results}

Identification of the FgPDK2 gene in F. graminearum The full sequence of FgPDK2 gene (FGSG_07381.3) was obtained from the $F$. graminearum genome sequence which was deposited in the FungiDB Institute Database (https://fungidb.org/fungidb/). There are three introns in the FgPDK2 gene. The first intron is located between the 150 th and 222th nucleotides and the length is $73 \mathrm{bp}$; the second intron is located between the 343rd and 393rd nucleotides and the length is $51 \mathrm{bp}$; the third intron is located between the 677th and 726th nucleotides and the length is $50 \mathrm{bp}$ (Fig. 1a). The deduced amino acid sequence of FgPDK2 from $F$. graminearum 
Table 1 Biological properties of the F. graminearum deletion mutant $\triangle F g P D K 2$, the complemented strain $\triangle F g P D K 2-C$, and the wild-type strain $\mathrm{PH}-1^{*}$

\begin{tabular}{|c|c|c|c|c|}
\hline \multirow[t]{2}{*}{ Strain } & \multicolumn{3}{|c|}{ Growth rate in vitro (mm/day) } & \multirow{2}{*}{$\begin{array}{l}\text { Conidia } \\
\text { produced } \\
\text { in vitro }(\times \\
\left.10^{5} / \mathrm{ml}\right)\end{array}$} \\
\hline & PDA & CM & MM & \\
\hline $\mathrm{PH}-1$ & $20.78 \pm 0.17 a$ & $21.22 \pm 0.45 a$ & $13.77 \pm 0.47 a$ & $1.77 \pm 0.42 \mathrm{a}$ \\
\hline$\Delta$ FgPDK2-1 & $5.36 \pm 0.33 b$ & $4.28 \pm 0.21 b$ & $3.11 \pm 0.32 b$ & $0 \mathrm{~b}$ \\
\hline$\Delta$ FgPDK2-2 & $6.18 \pm 0.14 b$ & $5.28 \pm 0.19 b$ & $3.27 \pm 0.23 b$ & $0 \mathrm{~b}$ \\
\hline$\triangle$ FgPDK2-C & $20.44 \pm 0.34 a$ & $21.11 \pm 0.17 a$ & $13.67 \pm 0.60 \mathrm{a}$ & $1.71 \pm 0.21 \mathrm{a}$ \\
\hline
\end{tabular}

*Values are the means ( \pm standard error) of three replicates. Means in a column followed by the same letter are not significantly different according to the LSD test at $P<0.05$

shares 79 and $70 \%$ identity with ToPDK2 of Tolypocladium ophioglossoides (KND93509.1) and NcPDK2 of Neurospora crassa (XP_961185.2), respectively (Fig. 1b), and FgPDK2 shares high similarity with other fungal PDK2 proteins (Fig. 1c).

\section{Involvement of FgPDK2 in mycelial morphogenesis and conidial formation of $F$. graminearum}

To determine the cellular function of $F g P D K 2$, we used the double-joint PCR approach to generate a gene replacement construct and transformed it into the $\mathrm{PH}-1$ strain. The two deletion mutants $\triangle$ FgPDK2 and the complementary mutant $\triangle$ FgPDK2-C were confirmed by PCR and Southern blot analyses (Supplemental data Fig. S1).

The deletion mutants lacking FgPDK2 ( $\triangle$ FgPDK21 and $\triangle \mathrm{FgPDK} 2-2)$ showed different growth patterns on three different kinds of medium (PDA, CM and $\mathrm{MM}$ ) compared with the wild-type strain $\mathrm{PH}-1$ and complemented mutant strains ( $\triangle$ FgPDK2-C) (Fig. 2a and Table 1). The deletion mutants $\triangle$ FgPDK2 grew significantly more slowly than the wild-type strain and did not produce any hyphae on these three kinds of media. The expression levels of the yellow pigment formation genes AurO and AurR2 were significantly downregulated in the deletion mutants compared with those in the wild-type strain $\mathrm{PH}-1$ and complemented mutant strains ( $\triangle$ FgPDK2-C), while the expression levels of the red pigmentformation genes AurJ and AurF were slightly upregulated (Fig. 2b). The results of microscopy examination showed that the deletion of FgPDK2 changed the morphology of aerial hyphae compared with that of the wild-type strain PH-1 (Fig. 3). In the process of asexual reproduction in F. graminearum, the ability to produce conidia is important. We found that the $\triangle$ FgPDK2 mutants failed to produce conidia compared to the wild-type and complemented strains (Table 1). These results showed that FgPDK2 is required for conidial formation and mycelial growth.

\section{Involvement of FgPDK2 in the cell membrane permeability of $F$. graminearum}

To ascertain the possible role of FgPDK2 in the cell membrane and cell wall integrity of $F$. graminearum, the deletion mutants $\triangle$ FgPDK2 were exposed to four stress-inducing reagents: osmotic stressors $(\mathrm{NaCl}$ and $\mathrm{KCl})$, cell membrane disrupter (SDS) and cell wall inhibitor (Congo red). Sensitivity to all those solutes was significantly greater in $\triangle$ FgPDK2 than in the parental strain $\mathrm{PH}-1$ or in the complemented strain. There were no significant changes in these cellular stresses between the FgPDK2-deficient mutants and the $\mathrm{PH}-1$ strain (Fig. 4). The results indicated that FgPDK2 is involved in the regulation of cell membrane and cell wall integrity in F. graminearum.

Endogenous reactive oxygen species (ROS) is an important indicator of the extent of cell membrane damage and here we used specific probe DCFH-DA to detect it. We found that ROS in the $\triangle \mathrm{FgPDK} 2$ mutants was increased compared to those in wild-type and complemented strains (Fig. 5). These results suggested that FgPDK2 may be an important regulator of the integrity of cell membranes and walls in the stress responses of F. graminearum.

\section{Involvement of FgPDK2 in virulence and DON biosynthesis of $F$. graminearum}

To explore the function of the FgPDK2 gene in pathogenicity, we inoculated the wild-type and mutant strains to the tomatoes. The mutants lacking $F g P D K 2$ showed a

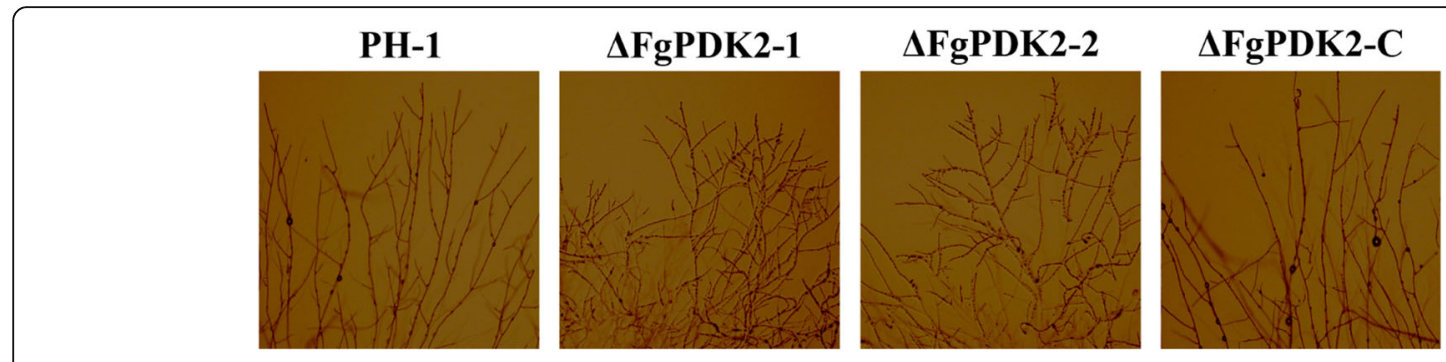

Fig. 3 Effect of FgPDK2 deletion on F. graminearum hyphae morphology by light micrographs 


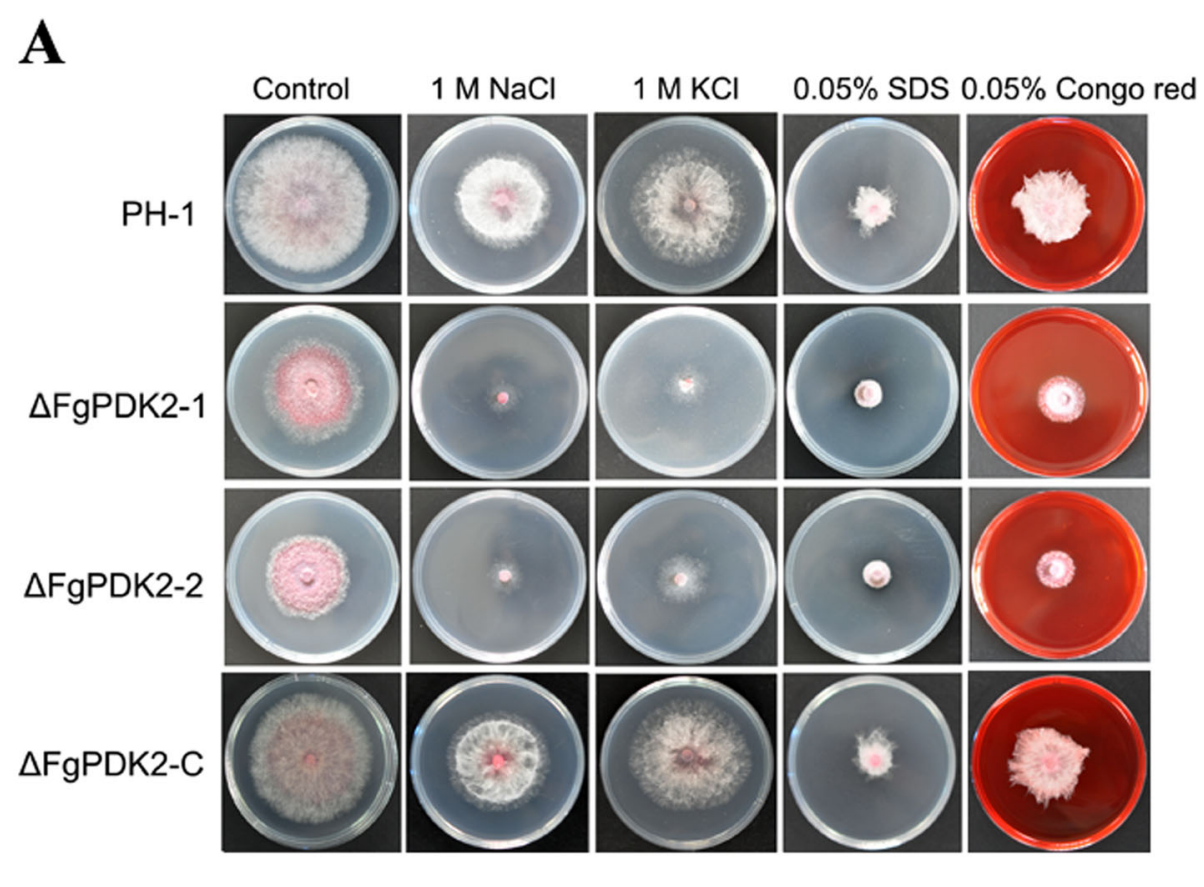

B

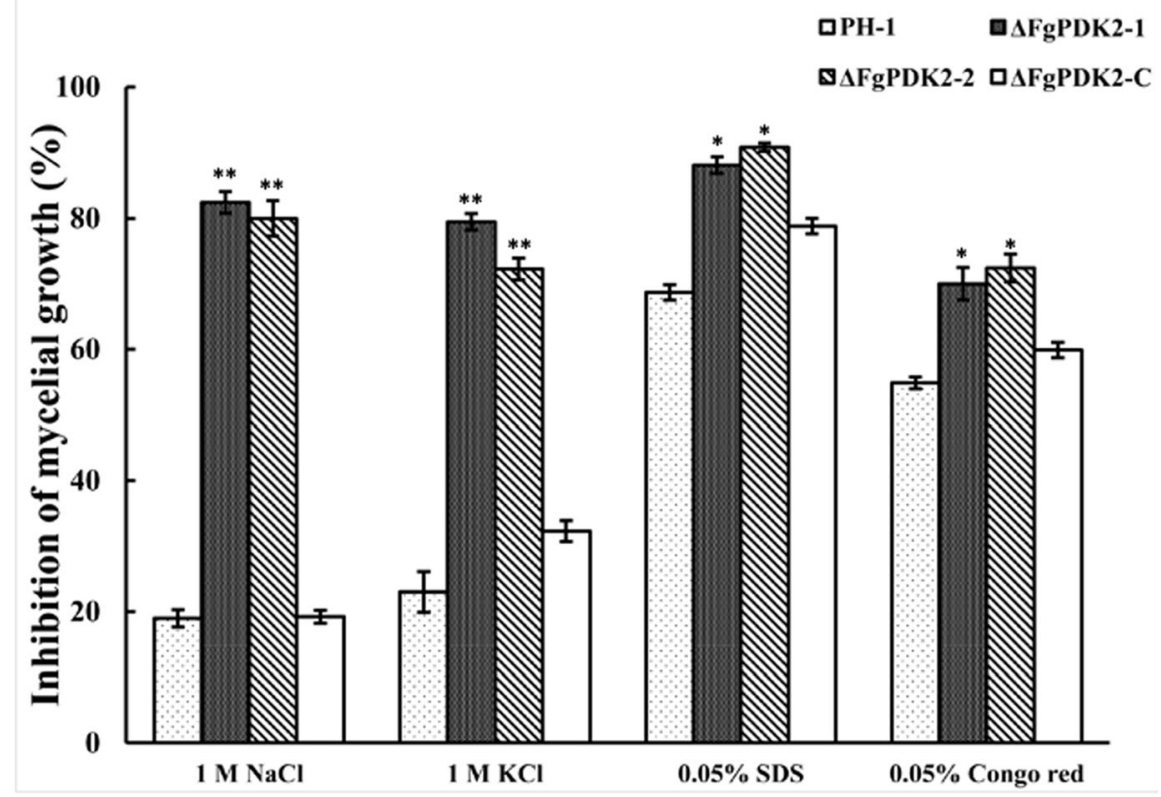

Fig. 4 Stress response of the wild-type strain PH-1, the FgPDK2 deletion mutant ( $\Delta$ FgPDK2), and the complemented strain ( $\Delta$ FgPDK2-C). The strains were grown in medium containing different chemicals as indicated. Then the photographs were taken $(\mathbf{a})$, and the inhibition of mycelial growth were measured (b). The error bars in each column represent the standard deviation (SD) from three independent experiments; ${ }^{*} P<0.05$ or ${ }^{* *} P<0.01$ by Student's t-test

loss of pathogenicity, whereas the wild-type and complemented strains caused serious lesions (Fig. 6).

DON is a kind of mycotoxin that is produced by $F$. graminearum, and it is a virulence factor that helps the fungus establish and spread. Here we compared the ability of DON biosynthesis between the wild-type and mutant strains. In this study, the deletion mutants produced significantly $(P<0.05)$ less DON than the wild-type and complemented strains (Fig. 7a).

The expression levels of two related genes of DON biosynthesis (Tri5 and Tri6) were significantly lower in the deletion mutants $(\triangle \mathrm{FgPDK} 2)$ than in the wild-type strain $\mathrm{PH}-1$ and complemented strain $(\triangle \mathrm{FgPDK} 2-\mathrm{C})$ (Fig. 7b). 


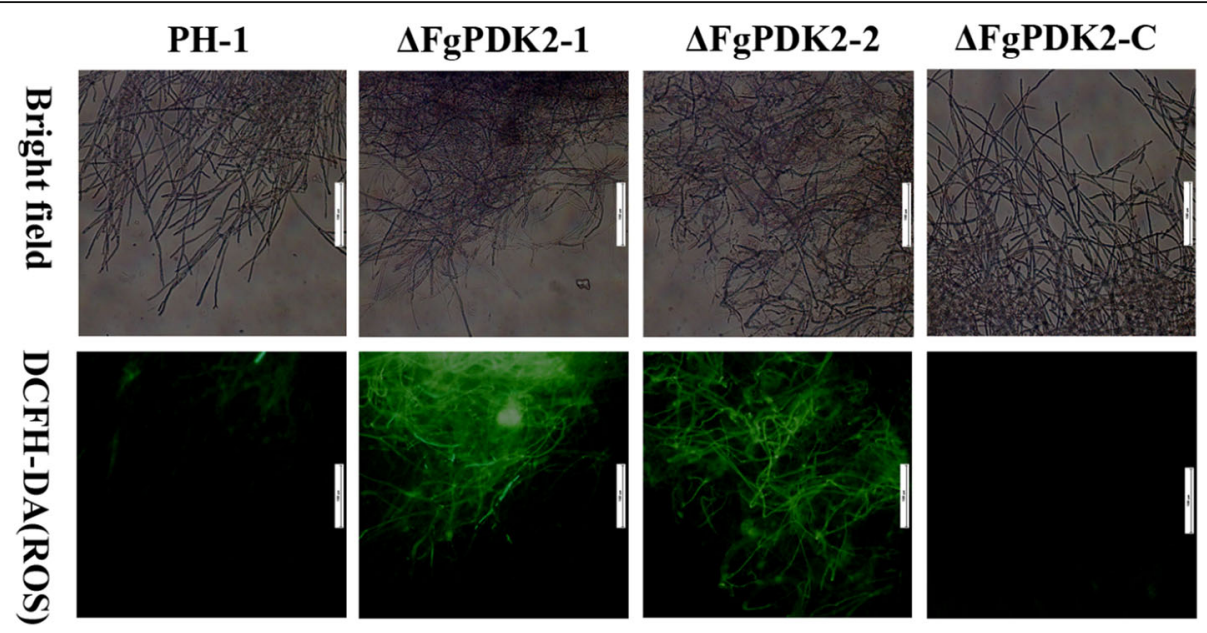

Fig. 5 The fluorescent staining of ROS in the mycelia of PH-1, the FgPDK2 deletion mutant ( $\triangle$ FgPDK2), and the complemented strain $(\Delta$ FgPDK2C). Mycelia were incubated with DCFH-DA followed by the fluorescent microscopic

\section{Discussion}

The mitochondrial pyruvate dehydrogenase complex $(\mathrm{PDH})$ represents the core of carbon metabolism and is connected with two main energy-generating pathways: glycolysis and the TCA cycle, which ultimately lead to ATP production (Zhang et al. 2014). PDH activity is controlled by PDHKs and PDHPs, and these two enzymes play an important role in fungal development, pathogenicity and enzyme secretion (de Assis et al. 2015; Gao et al. 2016; Brown et al. 2013). Thus, the PDK enzymes involved in basic growth and essential pathways are potential desirable candidates to develop fungicides.

In this study, the hypothetical pyruvate dehydrogenase kinase PDK2 cloned from $F$. graminearum was identified on the basis of high sequence homology with other PDKs and the presence of BCDHK domains. The conservation of the PDK protein indicates that the kinase enzyme is an important regulator of metabolism and that its mechanism has been conserved throughout evolution. In mammalian cells, phosphorylation is performed by one or more tissue-specific kinases (PDHK14) (Sugden et al. 2000). Pyruvate dehydrogenase complex (PDC) activity is crucial for maintaining blood glucose and ATP levels, which largely depend on the phosphorylation status determined by pyruvate dehydrogenase kinase (PDK) isoenzymes (Jeong et al. 2012). Malfunctions of the pyruvate dehydrogenase complex have been linked to several diseases, the most prominent of which is lactic acidosis (Brown et al. 1994). The PDK complex has been studied thoroughly in several eukaryotes including a number of mammals, but the situation in fungi is less clear. Therefore, this study investigated that defects in pyruvate dehydrogenase kinase affect fungal development in $F$. graminearum. For functional analysis of this gene, disruptions were generated as described in materials and methods section. Basically, the PDK gene was replaced by an HPH cassette. The FgPDK2 deletion mutants were viable but exhibited various defective developmental characteristics.

Studies have confirmed that the PDK complex of $S$. cerevisiae (both Pkp1 and the YGL059w product) is located in the mitochondria and that several amino acids (leucine, valine, isoleucine and arginine) occur (partly) in the mitochondria. In this study, the mutants exhibited substantial reductions in the formation of aerial mycelia and the production of conidia and perithecia. These changes evidently resulted in a reduced ability to infect hosts and cause disease. In the present study, the increased sensitivity to osmotic stresses mediated by the $\triangle$ FgPDK2 mutant suggested that $F g P D K 2$ is important for maintaining the integrity and stability of cell membranes. Under external stress conditions, cells produce a large amount of reactive oxygen species, which in turn cause lipid peroxidation damage in cell membrane. The PDK1 deletion of mutant of yeast showed high sensitivity to oxidative stress which was induced by hydrogen peroxide (Altintaş et al. 2015). This phenomenon was similar to that observed in the PDK2 deletion mutant in this study, which led to the accumulation of ROS.

Based on the foregoing, we may conclude that FgPDK2 may protect the cell membranes from stress by reducing reactive oxygen species. PDK2's ability to scavenge reactive oxygen species may be attributed to lower mitochondrial respiration (Newington et al. 2012).

The regulatory proteins directly associated with DON synthesis in F. graminearum are controlled by Tri genes (Gardiner et al. 2009). Among these genes, Tri5 and Tri6 are the most important related to DON biosynthesis (Proctor et al. 1995). In this study, the reduced 


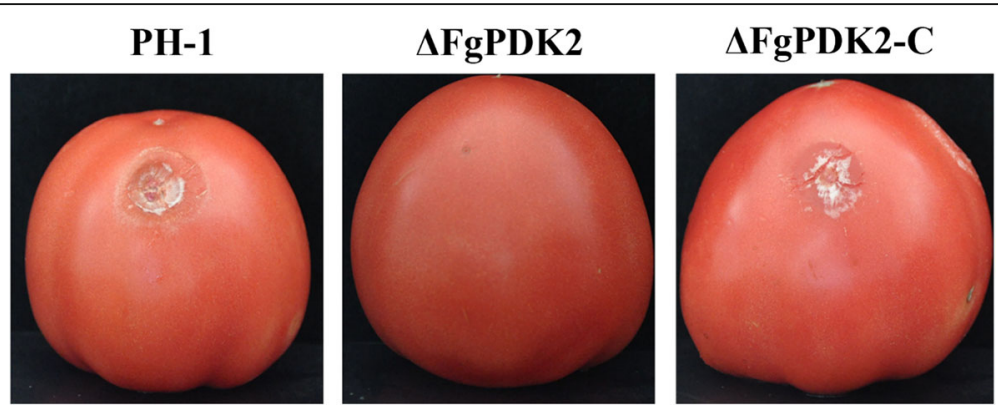

Fig. 6 Pathogenicity assays for F. graminearum strain PH-1, the FgPDK2 deletion mutant ( $\triangle$ FgPDK2) and the complemented strain ( $\triangle$ FgPDK2-C). Lesions caused by strains on tomato fruits. Lesions were photographed after 5 days at $100 \% \mathrm{RH}$ at $30^{\circ} \mathrm{C}$
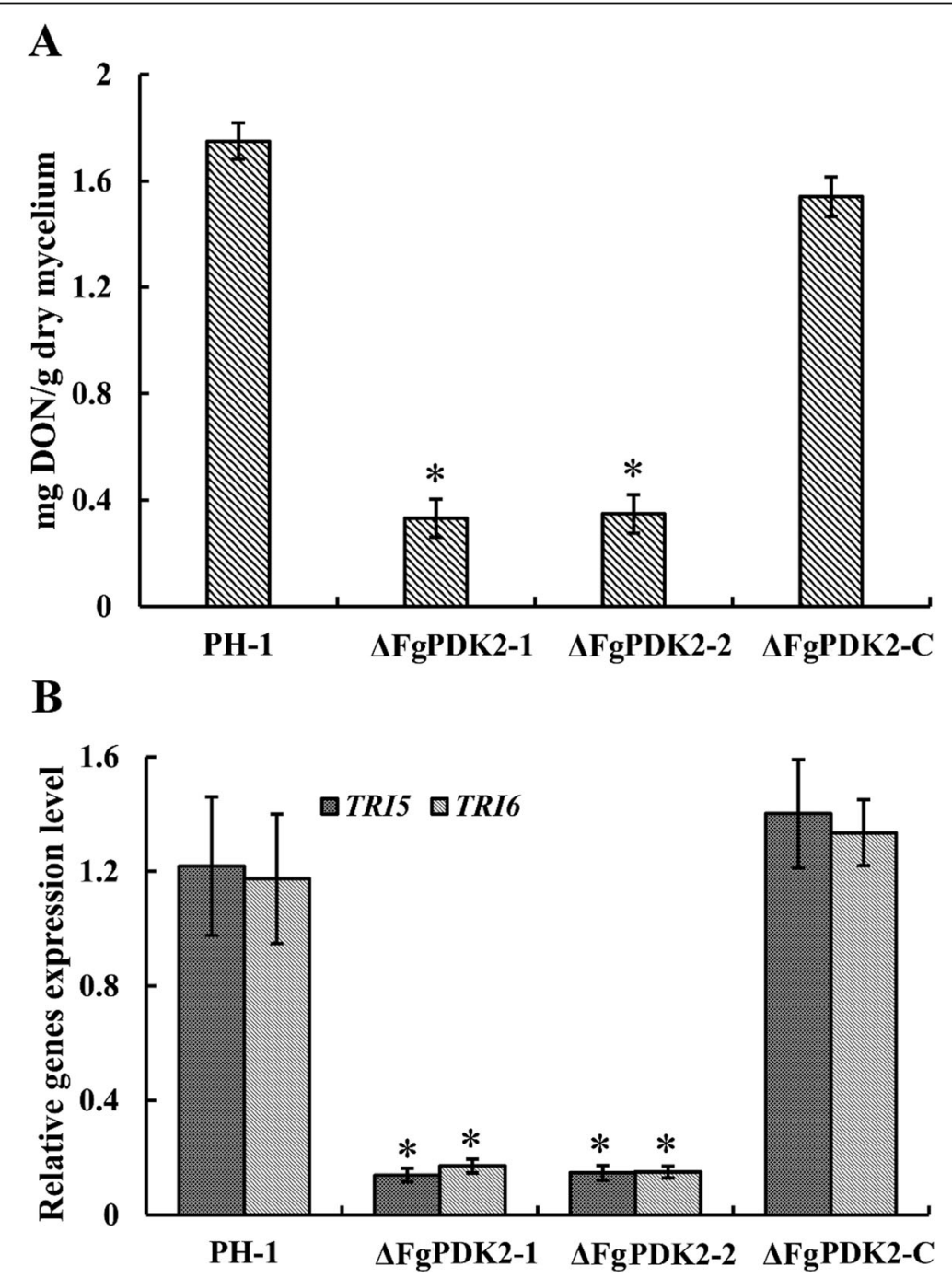

Fig. 7 Deoxynivalenol (DON) production (a) and relative expression of Tri5 and Tri6 (b) in PH-1, the FgPDK2 deletion mutant ( $\Delta$ FgPDK2), and the complemented strain ( $\triangle$ FgPDK2-C). The error bars in each column represent the standard deviation (SD) from three independent experiments; ${ }^{*} P<0.05$ by Student's t-test 
pathogenicity of the $F g P D K 2$ deletion mutants may also have resulted from low trichothecene production together with reduced expression of the Tri genes. The reason for these findings may be that the infectious capacity and regulatory activity of the mutant hyphae were reduced.

\section{Conclusions}

In summary, the function of PDK2 in the plant pathogen $F$. graminearum was characterized. It was found that $F g P D K 2$ is important in the growth of $F$. graminearum. Deletion of FgPDK2 can result in changes in mycelial morphogenesis, conidial formation and DON biosynthesis. Furthermore, FgPDK2 is also crucial for the integrity of the cell membrane and cell walls. Therefore, current study demonstrated the vital role of PDK2 in this phytopathogen and all the results procured may provide a potential molecular target for novel fungicides design to control the FHB and reduce toxin pollution, which is very important for wheat production and safety.

\section{Supplementary information}

Supplementary information accompanies this paper at https://doi.org/10. 1186/s43014-020-00025-x.

Additional file 1: Table S1. Oligonucleotide primers used in this study and their relevant characteristics. Fig. S1. Schematic representation of the FgPDK2deletion strategy.

\section{Abbreviations}

FHB: Fusarium Head Blight; DON: Deoxynivalenol; PDA: Potato Dextrose Agar; MBB: Mung Bean Broth; YEPD: Yeast Extract Peptone Dextrose; GYEP: Glucose Yeast Extract Peptone; PDK: Pyruvate Dehydrogenase Kinase; HPLC-MS/ MS: High-Performance Liquid Chromatography-Mass spectrometer/Mass spectrometer

\section{Acknowledgements}

Not applicable.

\begin{abstract}
Authors' contributions
Tao Gao was involved in data acquisition, draft and critical revision of article, and final approval; Dan He was involved in data analysis; Xin Liu and Fang Ji were involved in data acquisition and data consulting; Jianrong Shi and Jianhong Xu conceived and designed the paper. The authors read and approved the manuscript.

\section{Funding}

This work was supported by the National Natural Science Foundation (31772118, 31872914, 31901936), Jiangsu Agriculture Science and Technology Innovation Fund (CX(19)3004, CX(17)1003).
\end{abstract}

\section{Availability of data and materials}

Not applicable.

\section{Competing interests}

The authors declare no conflict of interest.

\section{Author details}

${ }^{1}$ Jiangsu Key Laboratory for Food Quality and Safety-State Key Laboratory Cultivation Base, Ministry of Science and Technology/Key Laboratory for Control Technology and Standard for Agro-product Safety and Quality, Ministry of Agriculture and Rural Affairs/Key Laboratory for Agro-product
Safety Risk Evaluation (Nanjing), Ministry of Agriculture and Rural Affairs/ Collaborative Innovation Center for Modern Grain Circulation and Safety/ Institute of Food Safety and Nutrition, Jiangsu Academy of Agricultural Sciences, Nanjing 210014, Jiangsu, China. ${ }^{2}$ School of Food and Biological Engineering, Jiangsu University, Zhenjiang, Jiangsu, China.

Received: 9 December 2019 Accepted: 4 May 2020

Published online: 26 May 2020

\section{References}

Altintaş, A., Martini, J., Mortensen, U. H., \& Workman, C. T. (2015). Quantification of oxidative stress phenotypes based on high-throughput growth profiling of protein kinase and phosphatase knockouts. FEMS Yeast Research, 16(1), 1-15.

Brown, G. K., Otero, L. J., LeGris, M., \& Brown, R. M. (1994). Pyruvate dehydrogenase deficiency. Journal of Medical Genetics, 31(11), 875-879.

Brown, N. A., De Gouvea, P. F., Krohn, N. G., Savoldi, M., \& Goldman, G. H. (2013). Functional characterisation of the non-essential protein kinases and phosphatases regulating Aspergillus Nidulans hydrolytic enzyme production. Biotechnology for Biofuels, 6(1), 1-17

Bruno, KS., Tenjo, F., Li, Lei., Hamer, J. E., Xu, J-R. (2004). Cellular Localization and Role of Kinase Activity of PMK1 in Magnaporthe grisea. Eukaryotic Cell, 3(6), $1525-1532$

Chen, Y., \& Zhou, M. G. (2009). Characterization of Fusarium Graminearum isolates resistant to both Carbendazim and a new fungicide JS399-19. Phytopathology, 99(4), 441-446.

de Assis, L. J., Ries, L. N. A., Savoldi, M., Dinamarco, T. M., Goldman, G. H., \& Brown N. A. (2015). Multiple phosphatases regulate carbon source-dependent germination and primary metabolism in Aspergillus Nidulans. G3: Genes, Genomes, Genetics, 5(5), 857-872.

Di Pietro, A., García-Maceira, F. I., Méglecz, E., \& Roncero, M. I. G. (2001). A MAP kinase of the vascular wilt fungus fusarium Oxysporum is essential for root penetration and pathogenesis. Molecular Microbiology, 39(5), 1140-1152.

Dickman, M. B., \& Yarden, O. (1999). Serine/threonine protein kinases and phosphatases in Filamentious fungi. Fungal Genetics and Biology, 26(2), 99117

Dong, F., Qiu, JB., Xu, JH., Yu, MZ., Wang, SF., Sun, Y., Zhang, GF., Shi, JR. (2016). Effect of environmental factors on Fusarium population and associated trichothecenes in wheat grain grown in Jiangsu province, China. International Journal of Food Microbiology, 230, 58-63.

Gao, T., Chen, J., \& Shi, Z. (2016). Fusarium Graminearum pyruvate dehydrogenase kinase 1 (FgPDK1) is critical for Conidiation, mycelium growth, and pathogenicity. PLoS One, 11(6), 1-19.

Gardiner, D. M., Osborne, S., Kazan, K., \& Manners, J. M. (2009). Low PH regulates the production of Deoxynivalenol by Fusarium Graminearum. Microbiology, 155(9), 3149-3156.

Jeong, J. Y., Jeoung, N. H., Park, K. G., \& Lee, I. K. (2012). Transcriptional regulation of pyruvate dehydrogenase kinase. Diabetes and Metabolism Journal, 36(5), 328-335.

Ji, F., Xu, J., Liu, X., Yin, X., \& Shi, J. (2014). Natural occurrence of Deoxynivalenol and Zearalenone in Wheat from Jiangsu Province, China. Food Chemistry, 157(1881), 393-397.

Kleter, G. A., \& Marvin, H. J. P. (2009). Indicators of emerging hazards and risks to food safety. Food and Chemical Toxicology, 47(5), 1022-1039.

Krause-Buchholz, U., Gey, U., Wünschmann, J., Becker, S., \& Rödel, G. (2006). YIL042C and YOR090c encode the kinase and phosphatase of the saccharomyces cerevisiae pyruvate dehydrogenase complex. FEBS Letters, 580(11), 2553-2560.

Kumar, S., Nei, M., Dudley, J., \& Tamura, K. (2008). MEGA: A biologist-centric software for evolutionary analysis of DNA and protein sequences. Briefings in Bioinformatics, 9(4), 299-306.

Liu, X., Jiang, Y., Zhang, Y., Yu, M., Jiang, H., Xu, J., \& Shi, J. (2019). Fgllv3a is crucial in branched-chain amino acid biosynthesis, vegetative differentiation, and virulence in fusarium Graminearum. Journal of Microbiology, 57, 694-703.

Mcmullen, M., Jones, R., Gallenberg, D., \& America, S. (1997). Scab of wheat and barley: a re-emerging disease of devastating impact. Plant Disease, 81(12), 1340-1348.

Newington, J. T., Rappon, T., Albers, S., Wong, D. Y., Jane Rylett, R., \& Cumming, R. C. (2012). Overexpression of pyruvate dehydrogenase kinase 1 and lactate dehydrogenase $\mathrm{a}$ in nerve cells confers resistance to amyloid $\beta$ and other toxins by decreasing mitochondrial respiration and reactive oxygen species production. Journal of Biological Chemistry, 287(44), 37245-37258. 
Papagianni, M. (2012). Recent advances in engineering the central carbon metabolism of industrially important bacteria. Microbial Cell Factories, 11, $1-13$.

Proctor, R. H., Hohn, T. M., McCormick, S. P., \& Desjardins, A. E. (1995). Tri6 encodes an unusual zinc finger protein involved in regulation of Trichothecene biosynthesis in fusarium Sporotrichioides. Applied and Environmental Microbiology, 61(5), 1923-1930.

Ries, L. N. A., de Assis, L. J., Rodrigues, F. J. S., Caldana, C., Rocha, M. C., Malavazi, I., Bayram, Ö., \& Goldman, G. H. (2018). The Aspergillus Nidulans pyruvate dehydrogenase kinases are essential to integrate carbon source metabolism. G3: Genes, Genomes, Genetics, 8(7), 2445-2463.

Roche, T. E., \& Hiromasa, Y. (2007). Pyruvate dehydrogenase kinase regulatory mechanisms and inhibition in treating diabetes, heart ischemia, and cancer. Cellular and Molecular Life Sciences, 64(7-8), 830-849.

Steensma H. Y., Tomaska, Lubomir., Reuven, Peter., Nosek, Jozef., Brandt, Raymond. (2008). Disruption of genes encoding pyruvate dehydrogenase kinases leads to retarded growth on acetate and ethanol inSaccharomyces cerevisiae. Yeast, 25(1), 9-19

Steensmays, H. Y. D. E. (1996). Pyruvate metabolism in. Yeast, 12, 1607-1633.

Sugden, M. C., \& Holness, M. J. (2011). The pyruvate carboxylase-pyruvate dehydrogenase Axis in islet pyruvate metabolism: Going round in circles? Islets, 3(6), 302-319.

Sugden, M. C., Langdown, M. L., Harris, R. A., \& Holness, M. J. (2000). Expression and regulation of pyruvate dehydrogenase kinase isoforms in the developing rat heart and in adulthood: Role of thyroid hormone status and lipid supply. Biochemical Journal, 352(3), 731-738.

Wang, C., Fan, J., Niu, C., Wang, C., Villaruz, A. E., Otto, M., \& Gao, Q. (2010). Role of Spx in biofilm formation of staphylococcus epidermidis. FEMS Immunology and Medical Microbiology, 59(2), 152-160.

Wang, J., Wang, H., Zhang, C., Wu, T., Ma, Z., \& Chen, Y. (2019). Phospholipid homeostasis plays an important role in fungal development, fungicide resistance and virulence in Fusarium Graminearum. Phytopathology Research $1(1), 1-12$.

Yu, J. H., Hamari, Z., Han, K. H., Seo, J. A., Reyes-Domínguez, Y., \& Scazzocchio, C. (2004). Double-joint PCR: A PCR-based molecular tool for gene manipulations in filamentous fungi. Fungal Genetics and Biology, 47(11), 973-981.

Zhang, S., Hulver, M. W., McMillan, R. P., Cline, M. A., \& Gilbert, E. R. (2014). The pivotal role of pyruvate dehydrogenase kinases in metabolic flexibility. Nutrition and Metabolism, 11(1), 1-9.

Zhang, Y. J., Fan, P. S., Zhang, X., Chen, C. J., \& Zhou, M. G. (2009). Quantification of Fusarium Graminearum in harvested grain by real-time polymerase chain reaction to assess efficacies of fungicides on fusarium head blight, Deoxynivalenol contamination, and yield of winter wheat. Phytopathology, 99(1), 95-100

Zheng, Z., Gao, T., Yu, Z., Hou, Y., Wang, J., \& Zhou, M. (2014). FgFim, a key protein regulating resistance to the fungicide JS399-19, asexual and sexual development, stress responses and virulence in Fusarium Graminearum. Molecular Plant Pathology, 15(5), 488-499.

\section{Publisher's Note}

Springer Nature remains neutral with regard to jurisdictional claims in published maps and institutional affiliations.

Ready to submit your research? Choose BMC and benefit from:

- fast, convenient online submission

- thorough peer review by experienced researchers in your field

- rapid publication on acceptance

- support for research data, including large and complex data types

- gold Open Access which fosters wider collaboration and increased citations

- maximum visibility for your research: over $100 \mathrm{M}$ website views per year

At BMC, research is always in progress.

Learn more biomedcentral.com/submissions 